\title{
Stability Analysis and Clinic Phenomenon Simulation of a Fractional-Order HBV Infection Model
}

\author{
Yongmei Su $\mathbb{D},{ }^{1}$ Sinuo Liu, ${ }^{1}$ Shurui Song, ${ }^{1}$ Xiaoke $\mathrm{Li}^{2},{ }^{2}$ and Yongan Ye $\mathbb{D}^{2}$ \\ ${ }^{1}$ School of Mathematics and Physics, University of Science and Technology Beijing, Beijing 100083, China \\ ${ }^{2}$ Institute of Liver Diseases, Beijing University of Chinese Medicine, \\ Dongzhimen Hospital Affiliated to Beijing University of Chinese Medicine, Beijing 100700, China \\ Correspondence should be addressed to Yongmei Su; suym71@ustb.edu.cn and Yongan Ye; yeyongan@vip.163.com
}

Received 6 August 2020; Revised 28 August 2020; Accepted 11 September 2020; Published 19 September 2020

Academic Editor: Songbai Guo

Copyright (c) 2020 Yongmei Su et al. This is an open access article distributed under the Creative Commons Attribution License, which permits unrestricted use, distribution, and reproduction in any medium, provided the original work is properly cited.

In this paper, a fractional-order HBV model was set up based on standard mass action incidences and quasisteady assumption. The basic reproductive number $R_{0}$ and the cytotoxic T lymphocytes' immune-response reproductive number $R_{1}$ were derived. There were three equilibrium points of the model, and stable analysis of each equilibrium point was given with corresponding hypothesis about $R_{0}$ or $R_{1}$. Some numerical simulations were also given based on HBeAg clinical data, and the simulation showed that there existed positive logarithmic correlation between the number of infected cells and HBeAg, which was consistent with the clinical facts. The simulation also showed that the clinical individual differences should be reflected by the fractional-order model.

\section{Introduction}

Viral infection is a major global problem, and mathematical models are an important tool for the study of biological phenomenon [1-5] and viral infectious disease [6-9] because they can help us to understand the dynamics of some infectious diseases and some chronic viral infections.

Mathematical models were also used to interpret experimental and clinical results in the fields of (anti-) HIV, HBV, and HCV infections [10-12]. These models were all set up with ordinary differential equation. In recent years, fractional differential equation models were often used in biology because the researchers found that the biological cell membranes have electron conductivity, which could be classified as a fractional-order model $[13,14]$. In addition, some biological models established by fractional differential equations have proved to be more advantageous than integers [14]. In particular, the biggest difference between the fractional-order model and the integer-order model is that the fractional-order model has the memory, while the characteristic of the immune response contains the memory [14].

So, when we discuss virus immune models, fractional mathematical models have become important tools.
Arafa et al. [14] proposed a fractional-order HIV infection model, Wang et al. [15] proposed a fractional-order HIV infection model, considering the logistic growth of the healthy CD4 cells, and Yan and Kou [16] had further proposed the following HIV model:

$$
\left\{\begin{array}{l}
D^{\alpha} x=\lambda-\mathrm{d} x+\rho x\left(1-x+\frac{y}{x_{\max }}\right)-\beta x v \\
D^{\alpha} y=\beta x v-a y \\
D^{\alpha} v=k y-\gamma v .
\end{array}\right.
$$

Within-host HBV models of fractional order were also discussed $[17,18]$, and the model in paper [17] was as follows:

$$
\left\{\begin{array}{l}
D^{\alpha} x=\lambda-\mathrm{d} x-\beta x v+\delta y \\
D^{\alpha} y=\beta x v-(a+\delta) y \\
D^{\alpha} v=k y-\gamma v
\end{array}\right.
$$

where $x, y$, and $v$ represent the concentration of uninfected hepatocytes cells, infected cells, and viruses, the death rate of 
them is $d, a$, and $\gamma$, reprectively, the uninfected hepatocytes are supposed to be produced at rate $\lambda, \beta$ is the infection rate at which the uninfected cell becomes infected, and the infected hepatocytes are cured by noncytolytic processes at a constant rate $\delta$ per cell. Based on model (2), Cardoso et al. [18] also disused a fractional model of hepatitis $B$ with drug therapy by representing $\beta$ and $k$ in model (2) with $(1-\rho) \beta$ and $(1-\mu) k$, in which $\rho$ and $\mu$ represent the drug efficacy.

It should be pointed out that the bilinear incidences $\beta x v$ were used to describe the infection between uninfected cells and virus in [14-18], but Min et al. changed it to standard incidence function $(\beta x v /(x+y))$ to describe the HBV infection model, which seemed more reasonable because it is independent of the number of total cells of liver [19]. On the other hand, Bartholdy et al. and Wodarz et al. [20] found that the turnover of free virus was much faster than that of infected cells, which means quasisteady assumption could be used, that is, the amount of free virus is simply proportional to the number of infected cells, so the number of infected cells $y$ can also be considered as a measure of virus load. Based on quasisteady assumption, Guo and Cai [21] discussed a HBV infection model by $(\beta x y /(x+y))$ instead of $(\beta x v /(x+y))$, but the model is integral order. On the other hand, model (2) does not include the immune cell, while the cytotoxic T lymphocyte (CTL) immune response after viral infection is universal and necessary to eliminate or control the disease.

Though fractional differential equations have proved to be a good choice to describe biological phenomenon, most discussions only focus on mathematic analysis and numerical simulation, and so far, almost no papers have used fractional-order models to explain clinic phenomenon about HBV. In this paper, based on the above discussion and quasisteady assumption, a HBV model was set up as follows:

$$
\left\{\begin{array}{l}
D^{\alpha} x=\lambda-\mathrm{d} x-\frac{\beta x y}{(x+y)}+\delta y, \\
D^{\alpha} y=\frac{\beta x y}{(x+y)}-a y-p y z-\delta y, \\
D^{\alpha} z=c y z-b z,
\end{array}\right.
$$

in which $0<\alpha<1$, the meanings of $x$ and $y$ are the same as those in model (2), $z$ represents the number of CTL, where the immune response is assumed to get stronger at a rate $c y z$ and decays exponentially at a rate $b z$, which is proportional to their current concentration, and the parameter $p$ expresses the efficacy of the nonlytic component.

This paper is organized as follows. In Section 2, some definitions and lemmas of fractional-order differential equation are cited. In Section 3, we mainly discuss the existence and uniqueness of positive solutions. The stability analysis is given in Section 4. The simulation is given in Section 5. This paper ends with a conclusion in Section 6 and discussion in Section 7.

\section{Primary Concept and Lemma}

It is known that fractional derivative has a variety of definitions $[17,22]$. In this paper, we used the Caputo fractional derivatives which are defined as follows.
Definition 1 (see [14]). The Caputo fractional derivatives of order $\alpha>0, n-1<\alpha<n, n \in N$, are defined as

$$
D^{\alpha} f(t)=\frac{1}{\Gamma(n-\alpha)} \int_{0}^{t} \frac{f^{(n)}(s)}{(t-s)^{\alpha+1-n}} \mathrm{~d} s,
$$

where $\Gamma(n-\alpha)=\int_{0}^{\infty} t^{n-\alpha} e^{-t} \mathrm{~d} t$.

Definition 2 (see [20]). The discriminant $D(f)$ of a polynomial

$$
f(x)=x^{n}+a_{1} x^{n-1}+a_{2} x^{n-2}+\cdots+a_{n},
$$

is defined by $D(f)=(-1)^{(n(n-1) / 2)} R\left(f, f^{\prime}\right)$, where $f^{\prime}$ is the derivative of $f . R\left(f, f^{\prime}\right)$ is the determinant of the corresponding $(2 n-1) \otimes(2 n-1)$ Sylvester matrix. The Sylvester matrix is formed by filling the matrix beginning with the upper left corner with the coefficients of $f(x)$ and then shifting down one row and one column to the right and filling in the coefficients starting there until they hit the right side. The process is then repeated for the coefficients of $f^{\prime}(x)$.

The following lemmas were useful to our arguments.

Lemma 1 (see [23]). For a fractional-order system:

$$
\left\{\begin{array}{l}
D^{\alpha} x(t)=f(t, x(t)) \\
x\left(t_{0}\right)=x_{0},
\end{array}\right.
$$

with $0<\alpha<1$ and $x \in R^{n}$, the equilibrium point of the system is locally asymptotically stable if all eigenvalues $\lambda_{i}$ of Jacobian matrix $J=(\partial f / \partial x)$ evaluated at the equilibrium point satisfy

$$
\left|\arg \left(\lambda_{i}\right)\right|>\frac{\alpha \pi}{2}
$$

Lemma 2 (see [24]). For the polynomial equation,

$$
P(\lambda)=\lambda^{n}+d_{1} \lambda^{n-1}+d_{2} \lambda^{n-2}+\cdots+d_{n}=0 .
$$

The conditions which make all the roots of (8) satisfy (7) are displayed as follows:

(i) For $n=1$, the condition is $d_{1}>0$

(ii) For $n=2$, the conditions are either Routh-Hurwitz conditions or

$$
\begin{aligned}
& d_{1}<0, \\
& 4 d_{2}>\left(d_{1}\right)^{2}, \\
&\left|\tan ^{-1}\left(\frac{\sqrt{4 d_{2}-\left(d_{1}\right)^{2}}}{d_{1}}\right)\right|>\frac{\alpha \pi}{2} .
\end{aligned}
$$

(iii) For $n=3$,

(a) If the discriminant $D(P)$ of $P(\lambda)$ is positive, then Routh-Hurwitz conditions are the 
necessary and sufficient conditions, that is, $d_{1}>0, d_{3}>0, d_{1} d_{2}>d_{3}$

(b) If $D<0, d_{1} \geq 0, d_{2} \geq 0$, and $d_{3}>0$, then (7) holds when $\alpha<(2 / 3)$

(c) If $D(P)<0, d_{1}<0$, and $d_{2}<0$, then (7) holds when $\alpha>(2 / 3)$

(d) If $D(P)<0, d_{1}>0, d_{2}>0$, and $d_{1} d_{2}=d_{3}$, then (7) holds for all $\alpha \in[0,1)$

Lemma 3 (see [25]). Assume that the vector function $f: R^{+} \times$ $R_{3} \longrightarrow R_{3}$ satisfies the following conditions:

(1) Function $f(t, x(t))$ is Lebesgue measurable with respect to $t$ on $R^{+}$

(2) Function $f(t, x(t))$ is continuous with respect to $x(t)$ on $R_{3}$

(3) $(\partial f(t, x(t)) / \partial x)$ is continuous with respect to $x(t)$ on $R_{3}$

(4) $\|f(t, X)\| \leq \omega+\lambda\|x\|, \forall t \in R^{+}, X \in R_{3}$

Here $\omega$ and $\lambda$ are two positive constants; then, initial value problems $D^{\alpha} X(t)=f(t, x(t)), X\left(t_{0}\right)=x_{0}, \alpha \in(0,1]$ have a unique solution.

Lemma 4 (see [26]). Let $m(t) \in C^{q}\left[\left(t_{0}, T\right], R\right]$ and assume that $\forall t_{1} \in\left(t_{0}, T\right], m\left(t_{1}\right)=0$ and $m(t)>0, t \in\left[t_{0}, t_{1}\right]$; then, $D^{q} m\left(t_{1}\right)<0$.

\section{The Existence and Uniqueness of Positive Solutions}

For the proof of the existence and uniqueness about the positive solution, we firstly prove that there exists a positively invariant region for system (3).

Let

$$
N(t)=x+y+\frac{p}{c} z
$$

and we have

$$
D^{\alpha} N(t)=\lambda-\mathrm{d} x-a y-\frac{p b}{c} z \leq \lambda-h\left(x+y+\frac{p}{c} z\right),
$$

in which $h=\min \{d, a, b\}$, so we have

$$
N(t) \leq\left(-\frac{\lambda}{h}+N(0)\right) E_{\alpha}\left(-h t^{\alpha}\right)+\frac{\lambda}{h} .
$$

Let $D=\{x+y+(p / c) z \leq(\lambda / h), x, y, z \geq 0\}$; it is easy to see that $D$ is a positively invariant region for model (3).

Theorem 1. For any initial condition in D, system (3) has a unique solution $X(t)=(x(t), y(t), z(t))^{T}$, and the solution will remain nonnegative for all $t \geq 0$.

Proof 1. Firstly, we prove the existence and uniqueness of the solution. We denote

$$
f(t, X)=\left(\begin{array}{c}
\lambda-d x-\left(\frac{\beta x y}{(x+y)}\right)+\delta y \\
\left(\frac{\beta x y}{x+y}\right)-a y-p y z-\delta y \\
c y z-b z
\end{array}\right) .
$$

Obviously $f(t, X)$ satisfies conditions (1)-(3) of Lemma 3 , and we only prove that system (3) satisfies the last condition (4) of Lemma 3. Let

$$
\begin{aligned}
\eta & =\left(\begin{array}{l}
\lambda \\
0 \\
0
\end{array}\right), \\
A_{1} & =\left(\begin{array}{ccc}
-d & \delta & 0 \\
0 & -a-\delta & 0 \\
0 & 0 & -b
\end{array}\right), \\
A_{2} & =\left(\begin{array}{ccc}
-\beta & 0 & 0 \\
\beta & 0 & 0 \\
0 & 0 & 0
\end{array}\right), \\
A_{3} & =\left(\begin{array}{lll}
0 & 0 & 0 \\
0 & -p & 0 \\
0 & 0 & 0
\end{array}\right), \\
A_{4} & =\left(\begin{array}{lll}
0 & 0 & 0 \\
0 & 0 & 0 \\
0 & 0 & c
\end{array}\right), \\
X(t) & =\left(\begin{array}{c}
x(t) \\
y(t) \\
z(t)
\end{array}\right) .
\end{aligned}
$$

Hence,

$$
\begin{aligned}
\|f(t, X)\| & =\left\|A_{1} X(t)+\frac{y(t)}{x(t)+y(t)} A_{2} X(t)+z(t) A_{3} X(t)+y(t) A_{4} X(t)+\eta\right\|, \\
& \leq\left\|A_{1} x(t)\right\|+\left\|A_{2} X(t)\right\|+\frac{c}{p} m\left\|A_{3} X(t)\right\|+m\left\|A_{4} X(t)\right\|+\|\eta\|, \\
& \leq\left(\left\|A_{1}\right\|+\left\|A_{2}\right\|+\frac{c}{p} m\left\|A_{3}\right\|+m\left\|A_{4}\right\|\right)\|X(t)\|+\|\eta\|,
\end{aligned}
$$


in which $m=(\lambda / h)$. By Lemma 3, system (3) has a unique solution.

We now prove the solution is nonnegative for all $t \geq 0$. From model (3), we know

$$
\begin{aligned}
& \left.D^{\alpha} x\right|_{x-0}=\lambda \geq 0, \\
& \left.D^{\alpha} y\right|_{y=0}=0, \\
& \left.D^{\alpha} y\right|_{z=0}=0 .
\end{aligned}
$$

By Lemma 4, the solution is nonnegative. In summary, the system has a unique nonnegative solution.

\section{Stable Analysis}

In this section, we will discuss the stability of model (3). This system always has an infection-free equilibrium $E_{0}=\left(x_{0}, 0,0\right)$, where $x_{0}=(\lambda / d)$.

The basic reproduction number is

$$
R_{0}=\frac{\beta}{(a+\delta)}
$$

When $R_{0}>1$, system (3) will have an immune-absence equilibrium $E_{1}=\left(x_{1}, y_{1}, 0\right)$, where

$$
\begin{aligned}
& x_{1}=\frac{\lambda(a+\delta)}{(a+\delta) d+a(\beta-a-\delta)}, \\
& y_{1}=\frac{\lambda(\beta-a-\delta)}{(a+\delta) d+a(\beta-a-\delta)},
\end{aligned}
$$

which means the uninfected cells and infected cells coexist but the immune response is not activated yet, that is, $c y_{1}<b$. Further, we will give the immune-response reproductive number $R_{1}=\left(c y_{1} / b\right)$ when $R_{1}>1$, that is, $c y_{1}>b$, which means immune response is activated. So, when $R_{1}>1$, there is another immune-response equilibrium $E_{2}=\left(x_{2}, y_{2}, z_{2}\right)$, where

$$
\begin{aligned}
& x_{2}=\frac{\Delta+\sqrt{\Delta^{2}+4 d\left(\delta y_{2}^{2}+\lambda y_{2}\right)}}{2 d c}, \\
& y_{2}=\frac{b}{c}, \\
& z_{2}=\frac{\beta c x_{2}}{p\left(c x_{2}+b\right)}-\frac{(a+\delta)}{p},
\end{aligned}
$$

in which $\Delta=\lambda-d y_{2}-\beta y_{2}+\delta y_{2}$. Note that $x_{2}, y_{2}$, and $z_{2}$ must be positive, and we can prove $z_{2}>0$ holds only when $R_{1}>1$. Hence, $E_{2}$ exists if and only if $R_{1}>1$.

Now, we introduce the main theorem.

Theorem 2. For system (3), if $R_{0}<1$, the equilibrium $E_{0}$ is globally asymptotically stable. If $R_{0}>1$, the equilibrium $E_{0}$ is unstable.

Proof 2. Jacobian matrix $J$ at $E_{0}$ is

$$
J=\left(\begin{array}{ccc}
\lambda+d & \beta-\delta & 0 \\
0 & \lambda+a+\delta-\beta & 0 \\
0 & 0 & \lambda+b
\end{array}\right) .
$$

The characteristic equation for the infection-free equilibrium $E_{0}$ is given as follows:

$$
(\lambda+d)(\lambda+a+\delta-\beta)(\lambda+b)=0 .
$$

We can see that the characteristic roots $\lambda_{1}=-d<0, \lambda_{2}=-b<0$, and when $R_{0}<1, \lambda_{3}=\beta-a-\delta<0$, all the characteristic roots satisfied $\left|\arg \lambda_{i}\right|=\pi>\alpha(\pi / 2)$, which shows that $E_{0}$ is locally asymptotically stable by Lemma 1. If $R_{0}>1,\left|\arg \lambda_{3}\right|=0<\alpha(\pi / 2), E_{0}$ is unstable.

Let

$$
V(x)=y+\frac{p}{c} z
$$

and we have

$$
\begin{aligned}
D^{\alpha} V & =D^{\alpha} y+\frac{p}{c} D^{\alpha} z, \\
& =\left(\frac{\beta x y}{(x+y)}\right)-a y-p y z-\delta y+\frac{p}{c}(c y z-b z), \\
& =\left(\frac{x}{x+y}\right) * \beta y-(a+\delta) y-\frac{p b}{c} z, \\
& \leq \beta y-(a+\delta) y=(a+\delta) y\left(R_{0}-1\right) .
\end{aligned}
$$

Since $\quad R_{0}<1$, we have $D^{\alpha} V \leq 0$. Let $M=\left\{(x, y, z) \in D, D^{\alpha} V=0\right\} ;$ obviously, $M \subset\{(x, y, z) \in$ $D, y=0\}$. Let $E$ be the largest positively invariant subset of $M$; obviously, $E$ is nonempty since $((\lambda / d), 0,0) \in E$. Let $(x(t)$, $y(t), z(t))$ be the solution of system (3) with initial value $\left(x_{0}, y_{0}, z_{0}\right) \in E$; then, $y(t)=0$. By the first and the third equations of (3), we have

$$
\left\{\begin{array}{l}
x(t)=\left(-\frac{\lambda}{d}+x(0)\right) E_{\alpha}\left(-\mathrm{d} t^{\alpha}\right)+\frac{\lambda}{d}, \\
z(t)=z(0) E_{\alpha}\left(-b t^{\alpha}\right),
\end{array}\right.
$$

and when, $x(t) \longrightarrow(\lambda / d), z(t) \longrightarrow 0$, by the invariance of $E, x(t)=(\lambda / d), z(t)=0$. By $t \longrightarrow$ othe Lyapunov-LaSalle theorem [26], when $t \longrightarrow \infty$, all solutions in the set $D$ approach $E_{0}$. Noting that $E_{0}$ is locally asymptotically stable, the infection-free equilibrium $E_{0}$ is globally asymptotically stable.

Theorem 3. For system (3), when $R_{0}>1$,

(1) The equilibrium $E_{1}$ is locally asymptotically stable if $R_{1}<1$

(2) If $R_{1}>1$, the equilibrium $E_{1}$ is unstable

Proof 3. The characteristic equation for the equilibrium $E_{1}$ is given as 


$$
\operatorname{det}\left(\begin{array}{ccc}
\lambda+d+\frac{\beta y_{1}^{2}}{\left(x_{1}+y_{1}\right)^{2}} & \frac{\beta x_{1}^{2}}{\left(x_{1}+y_{1}\right)^{2}}-\delta & 0 \\
-\frac{\beta y_{1}^{2}}{\left(x_{1}+y_{1}\right)^{2}} & \lambda-\frac{\beta x_{1}^{2}}{\left(x_{1}+y_{1}\right)^{2}}+a+\delta & p y_{1} \\
0 & 0 & \lambda-c y_{1}+b
\end{array}\right)=0 \text {, }
$$

that is,

$$
\left(\lambda-c y_{1}+b\right)\left[\lambda^{2}+\left(a+\delta+d+\frac{\beta\left(y_{1}^{2}-x_{1}^{2}\right)}{\left(x_{1}+y_{1}\right)^{2}}\right) \lambda+(a+\delta) d+\frac{\beta\left(a y_{1}^{2}-\mathrm{d} x_{1}^{2}\right)}{\left(x_{1}+y_{1}\right)^{2}}\right]=0 .
$$

We can see $\lambda_{1}=c y_{1}-b$, and when $R_{1}<1, \lambda_{1}$ is negative

We let and $\left|\arg \lambda_{1}\right|=\pi>\alpha(\pi / 2)$.

Next, we consider the following equation:

$\lambda^{2}+\left(a+\delta+d+\frac{\beta\left(y_{1}^{2}-x_{1}^{2}\right)}{\left(x_{1}+y_{1}\right)^{2}}\right) \lambda+(a+\delta) d+\frac{\beta\left(a y_{1}^{2}-\mathrm{d} x_{1}^{2}\right)}{\left(x_{1}+y_{1}\right)^{2}}=0$.

$$
\begin{aligned}
& B=a+\delta+d+\frac{\beta\left(y_{1}^{2}-x_{1}^{2}\right)}{\left(x_{1}+y_{1}\right)^{2}}=a+\delta+d+\beta \frac{\left(y_{1}-x_{1}\right)}{\left(x_{1}+y_{1}\right)}=(a+\delta)\left(R_{0}-1\right)+d, \\
& C=(a+\delta) d+\frac{\beta\left(a y_{1}^{2}-\mathrm{d} x_{1}^{2}\right)}{\left(x_{1}+y_{1}\right)^{2}}=\frac{d(a+\sigma)(a+\delta)\left(R_{0}-1\right)+a(a+\delta)^{2}\left(R_{0}-1\right)^{2}}{\beta} .
\end{aligned}
$$

Since $R_{0}>1$, we can see $B>0, C>0$; hence, (27) has two negative real roots, and we denote them by $\lambda_{2}$ and $\lambda_{3}$ $\left(\left|\arg \lambda_{2}\right|=\pi>\alpha(\pi / 2)\right.$ and $\left.\left|\arg \lambda_{3}\right|=\pi>\alpha(\pi / 2)\right)$, so when $R_{1}<1$, the equilibrium $E_{1}$ is locally asymptotically stable. When $R_{1}>1, \lambda_{1}$ is positive, and $\left|\arg \lambda_{1}\right|=0<\alpha(\pi / 2), E_{1}$ is unstable.

Finally, we discuss the local stability of the immuneresponse equilibrium state $E_{2}$, and the Jacobian matrix at $E_{2}$ is given by

$$
J=\left(\begin{array}{ccc}
-d-\frac{\beta y_{2}^{2}}{\left(x_{2}+y_{2}\right)^{2}} & -\frac{\beta x_{2}^{2}}{\left(x_{2}+y_{2}\right)^{2}}+\delta & 0 \\
\frac{\beta y_{2}^{2}}{\left(x_{2}+y_{2}\right)^{2}} & \frac{\beta x_{2}^{2}}{\left(x_{2}+y_{2}\right)^{2}}-a-\delta-p z_{2} & -p y_{2} \\
0 & -c z_{2} & c y_{2}-b
\end{array}\right)
$$

The corresponding characteristic equation is

$$
P(\lambda)=\lambda^{3}+a_{1} \lambda^{2}+a_{2} \lambda+a_{3}=0,
$$

where

$$
\begin{aligned}
a_{1} & =d+a+\delta+p z_{2}+\frac{\beta y_{2}^{2}-x_{2}^{2}}{\left(x_{2}+y_{2}\right)^{2}}, \\
& =d+\frac{\beta x_{2}}{x_{2}+y_{2}}+\frac{\beta\left(y_{2}-x_{2}\right)}{x_{2}+y_{2}}, \\
& =d+\frac{\beta y_{2}}{x_{2}+y_{2}}>0, \\
a_{2} & =\frac{\beta y_{2}^{2}}{\left(x_{2}+y_{2}\right)^{2}}\left(a+p z_{2}\right)+p b z_{2}+d\left(a+\delta+p z_{2}\right)-\frac{\beta x_{2}^{2} d}{\left(x_{2}+y_{2}\right)^{2}}, \\
& =\frac{\beta y_{2}^{2}}{\left(x_{2}+y_{2}\right)^{2}}\left(a+p z_{2}\right)+p b z_{2}+d \frac{\beta x_{2}}{x_{2}+y_{2}}-\frac{\beta x_{2}^{2} d}{\left(x_{2}+y_{2}\right)^{2}}, \\
& =\frac{\beta y_{2}^{2}}{\left(x_{2}+y_{2}\right)^{2}}\left(a+p z_{2}\right)+p b z_{2}+\beta d \frac{x_{2} y_{2}}{\left(x_{2}+y_{2}\right)^{2}}>0, \\
a_{3} & =p b z_{2}\left(d+\frac{\beta y_{2}^{2}}{\left(x_{2}+y_{2}\right)^{2}}\right)>0 .
\end{aligned}
$$

It is easy to verify that 


$$
a_{1} a_{2}>p b z_{2}\left(d+\frac{\beta y_{2}}{x_{2}+y_{2}}\right)>p b z_{2}\left(d+\frac{\beta y_{2}^{2}}{\left(x_{2}+y_{2}\right)^{2}}\right)=a_{3}
$$

Based on Definition 2, we obtain

$$
\begin{aligned}
D(P) & =-\left|\begin{array}{ccccc}
1 & a_{1} & a_{2} & a_{3} & 0 \\
0 & 1 & a_{1} & a_{2} & a_{3} \\
3 & 2 a_{1} & a_{2} & 0 & 0 \\
0 & 3 & 2 a_{1} & a_{2} & 0 \\
0 & 0 & 3 & 2 a_{1} & a_{2}
\end{array}\right| \\
& =18 a_{1} a_{2} a_{3}+\left(a_{1} a_{2}\right)^{2}-4 a_{1}^{2}-4 a_{2}^{2}-27 a_{3}^{2} .
\end{aligned}
$$

By Lemma 2, we have the following theorem.

Theorem 4. For system (3), when $R_{1}>1$,

(1) If the discriminant $D(P)$ of $P(\lambda)$ is positive, the equilibrium $E_{2}$ is locally asymptotically stable for $0<\alpha<1$

(2) If $D(P)<0$, then the equilibrium $E_{2}$ is locally asymptotically stable for $0<\alpha<(2 / 3)$

\section{Numerical Simulation}

5.1. Simulation of the Immune-Response Equilibrium. We first simulate the stability of $E_{2}$, and the locally stable condition of $E_{2}$ is given by Theorem 4 . The parameters we used are listed in Table 1 which can make $R_{1}=578.5076>1$, $D(P)=-0.1296<0$ hold. We choose $\alpha=0.55, \alpha=0.6$ and $\alpha=0.65$, which satisfy $0<\alpha<(2 / 3)$, and the simulation is shown in Figure 1. The dynamic routes of uninfected and infected cells are shown in Figures 1(a) and 1(b), and the CTL cells are shown in Figure 1(c). The simulation shows that the immune-response equilibrium $E_{2}$ is locally asymptotically stable for $0<\alpha<(2 / 3)$, which is consistent with the conclusion of Theorem 4 .

5.2. Simulation of Correlation between HBeAg and Infected Cells. A positive correlation was found between log-HBV DNA and log-HBeAg in [27], that is, the higher the HBeAg, the higher the HBV DNA load. Since by quasisteady assumption, the amount of free virus is simply proportional to the number of infected cells, the number of infected cell should also be positive logarithmic correlative with $\mathrm{HBeAg}$. Since we could not get the specific number of infected cells from clinic, $\mathrm{HBeAg}$ can be quantified. In the following part, we will testify the positive correlation between the infected cell and the HBeAg based on the clinical data of HBeAg by numerical simulation. We use four $\mathrm{CHB}$ patients' $\mathrm{HBeAg}$ clinical data of 108 weeks with the treatment of entecavir from Dongzhimen Hospital; two patients were from successful-treatment group, and the other two patients were from unsuccessful-treatment group; the data are shown in Tables 2 and 3.
By Theorem 2, the infection-free equilibrium $E_{0}=\left(x_{0}, 0,0\right)$ is asymptotically stable if $R_{0}<1$. When simulating the successful-treatment group, the parameters we choose should make $R_{0}<1$. Considering the biological significance of the parameters, we choose the parameters in Table 4 as follows.

A human liver contains approximately $2 \times 10^{11}$ hepatocytes [28], and a patient has a total of about $3000 \mathrm{ml}$ plasma. Usually, clinical testing quantification is based on one millilitre. Consequently, we can assume that $(\lambda / d) \approx\left(2 \times 10^{11} / 3000\right)$. Since the half-life of a hepatocyte is about half a year, we choose $d=0.00379$ similar to [28]. The half-life of CTL is about 77 days [29], so we choose $b=0.009$. Other parameters were chosen by simulation. The parameters in Table 4 can make $R_{0}=0.045<1$ hold. We give three simulation results with $\alpha=1, \alpha=0.9$, and $\alpha=0.8$; the simulations are shown in Figure 2. The dynamic routs of uninfected cells and CTL cells are shown in Figures 2(a) and 2(b), and the HBeAg and infected cells are shown in Figure 2(c). The simulation shows that the infection-free equilibrium $E_{0}$ is asymptotically stable with different $0<\alpha<1$ which is consistent with the conclusion of Theorem 2 , but when $\alpha=1$, the dynamic route is obviously different. From the simulation in Figure 2(c), we can also observe the positive correlation between log-infected cells and logHBeAg for different $\alpha<1$, which is consistent with clinical fact. On the other hand, from the clinical data of $\mathrm{HBeAg}$, we can see that the change rate was different between different patient, though they have same change trend, which shows that individual differences do exist in clinical therapy even with the same medicine and the same dose; the clinical phenomenon may be explained by our model to some extent. From the simulation, we can see that the larger the $\alpha$, the slower the decrease rate of infected cells and CTL in the former stage, but the faster the decrease rate in the later stage; the individual difference may be reflected by different $\alpha$, and the fractional-order model should be a good tool to describe HBV infection.

When we simulate the unsuccessful-treatment group in Table 3, the patients' ALT level was lower than normal standard, so we can think that the patient has not yet developed an immune response to the hepatitis $B$ virus. When $R_{0}>1$, there exists the equilibrium $E_{1}$ without immune activities, so we choose the immune parameter $b$ larger than that in Table 4 and the immune parameter $c$ smaller than that in Table 4 . The death rate of infected cells should decrease without immune activities, so we choose parameter $a$ which is smaller than that in Figure 2. Since $E_{1}$ is asymptotically stable, if $R_{1}<1$, the parameter we choose in Table 5 can make $R_{0}=2.4253>1$ and $R_{1}=0.1351<1$ hold. We also used $\alpha=0.9, \alpha=0.85$, and $\alpha=0.8$ to simulate, and the simulation is shown in Figure 3. The dynamic routes of uninfected cells and CTL cells are shown in Figures 3(a) and 3(b), and the $\mathrm{HBeAg}$ and infected cells are shown in Figure 3(c). From the simulation, the dynamic routes of uninfected cells, infected cells, and CTL cells show that the immune-absence equilibrium $E_{1}$ is asymptotically stable with different $\alpha$ which is consistent with the conclusion of Theorem 3. From the simulation, there still exists the same change trend but 
TABle 1: Parameters in Figure 1.

\begin{tabular}{lccc}
\hline Parameter & Value & Parameter & Value \\
\hline$\lambda$ & 10 & $c$ & 0.051 \\
$\beta$ & 0.01 & $d$ & 0.18 \\
$a$ & 0.003 & $p$ & 0.006 \\
$b$ & 0.011 & $\delta$ & 0.000000018 \\
\hline
\end{tabular}
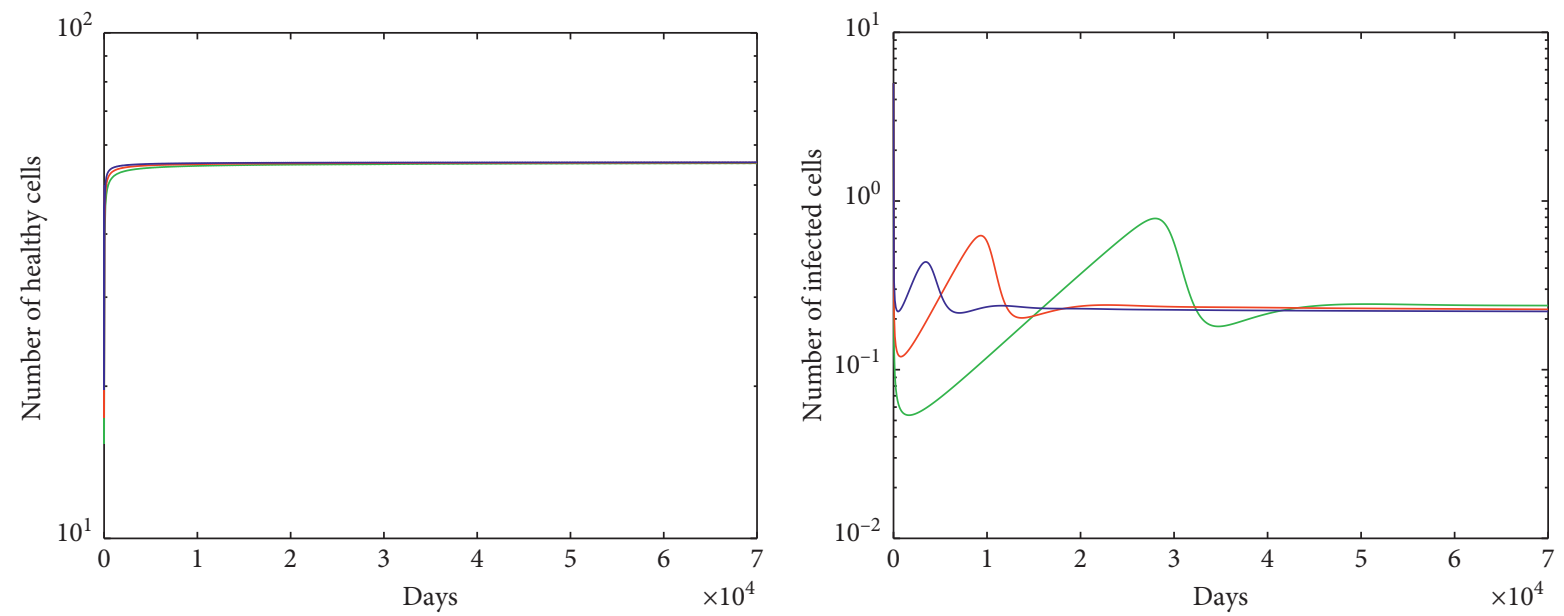

$\begin{aligned} \alpha & =0.55 \\ \alpha & =0.6 \\ \alpha & =0.65\end{aligned}$

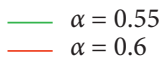

$\begin{aligned} \alpha & =0.6 \\ \alpha & =0.65\end{aligned}$

(a)

(b)

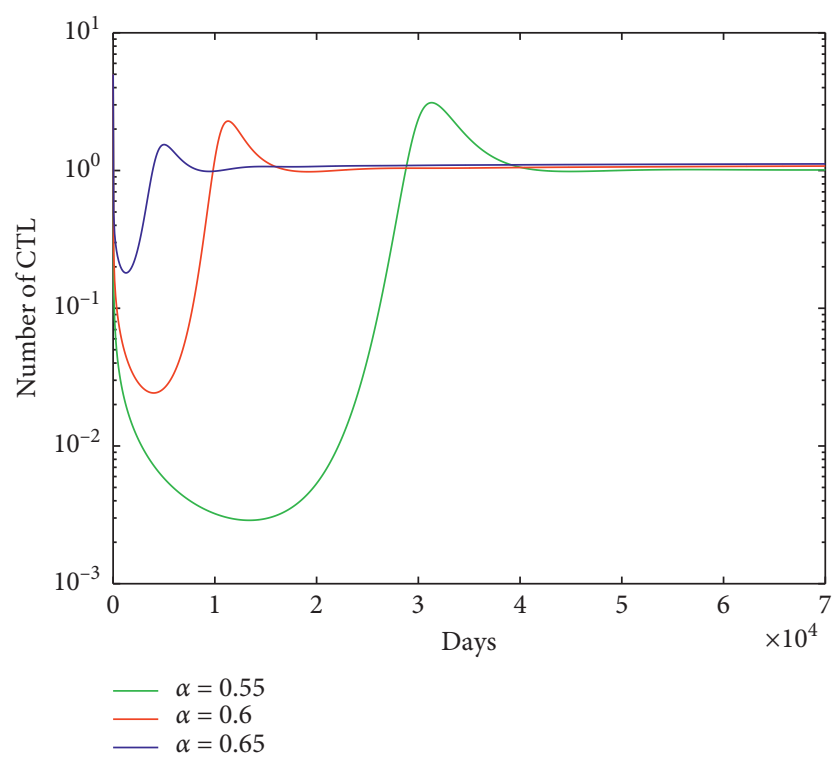

(c)

FIgURE 1: Simulation results of immune-response equilibrium $E_{2}$ with different $\alpha$. 
TABLE 2: HBeAg data of successful-treatment group.

\begin{tabular}{lccccccccccc}
\hline & 0 & 12 & 24 & 36 & 48 & 60 & 72 & 84 & 96 & 108 (week) \\
\hline I & 819.51 & 34.55 & 12.44 & 4.23 & 1.12 & 0.61 & 0.44 & 0.38 & 0.38 & 0.44 \\
II & 874.31 & 12.15 & 1.88 & 1.65 & 1.15 & 0.82 & 0.36 & 0.4 & 0.37 & 0.31 \\
\hline
\end{tabular}

TABLE 3: HBeAg data of unsuccessful-treatment group.

\begin{tabular}{lcccccccccc}
\hline & 0 & 12 & 24 & 36 & 48 & 60 & 72 & 84 & 96 & 108 (week) \\
\hline I & 36.79 & 138.6 & 221.36 & 314.88 & 393.78 & 391.75 & 378.63 & 411.61 & 407.91 & 342.63 \\
II & 44.11 & 85.05 & 140.27 & 141.82 & 125.86 & 142.63 & 158.75 & 194.48 & 0.6 & 243.72 \\
\hline
\end{tabular}

Table 4: Parameters in Figure 2.

\begin{tabular}{lccc}
\hline Parameter & Value & Parameter & Value \\
\hline$\lambda$ & $2.53 e+005$ & $c$ & $0.31 e-007$ \\
$\beta$ & $6.9 e-004$ & $a$ & $4 * 3.79 e-003$ \\
$d$ & $3.79 e-003$ & $p$ & $1.5 e-004$ \\
$b$ & $0.9 e-002$ & $\delta$ & $1.9 e-004$ \\
\hline
\end{tabular}

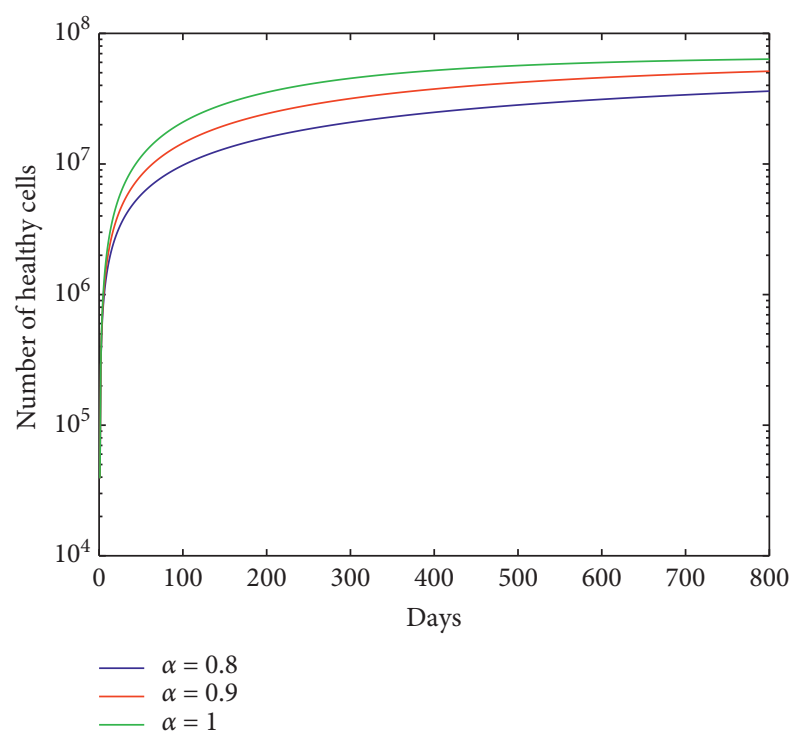

(a)

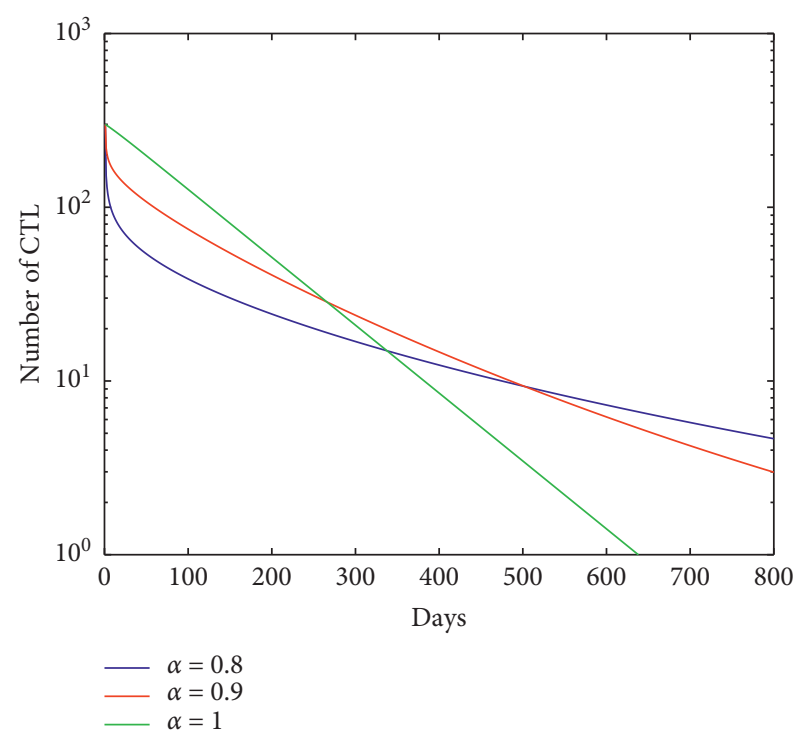

(b)

Figure 2: Continued. 


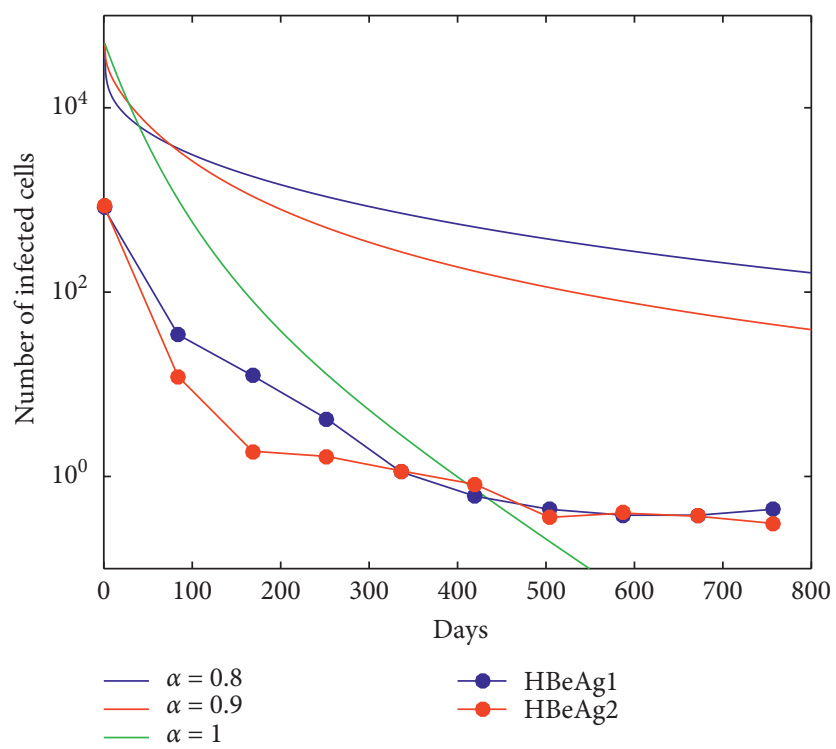

(c)

FIGURE 2: Simulation results of successful-treatment group.

TABle 5: Parameters in Figure 3.

\begin{tabular}{lccc}
\hline Parameter & Value & Parameter & Value \\
\hline$\lambda$ & $2.53 e+005$ & $c$ & $0.31 e-009$ \\
$\beta$ & $(6.9 e-003) * 2$ & $a$ & $3.79 e-003$ \\
$d$ & $3.79 e-003$ & $p$ & $1.5 e-004$ \\
$b$ & 0.9 & $\delta$ & $1.9 e-004$ \\
\hline
\end{tabular}

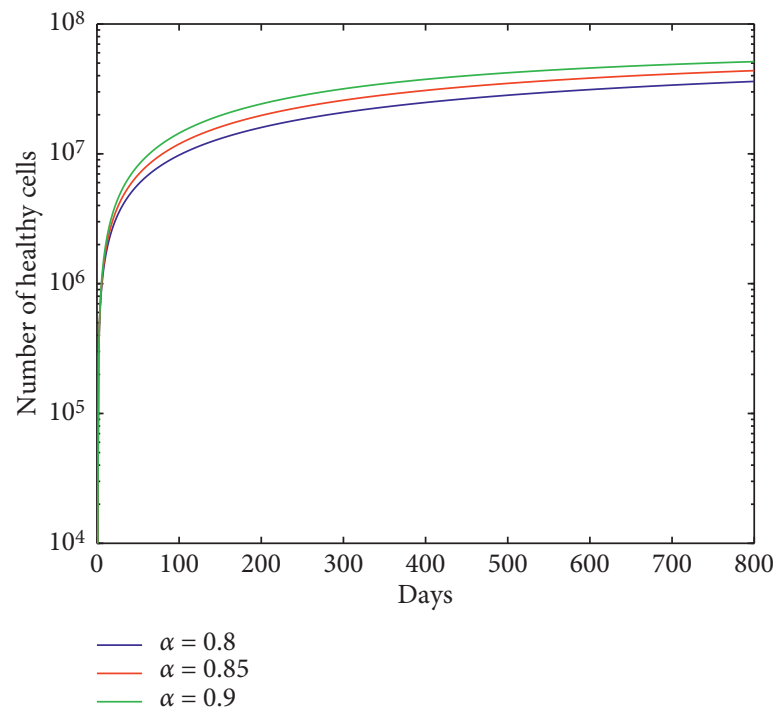

(a)

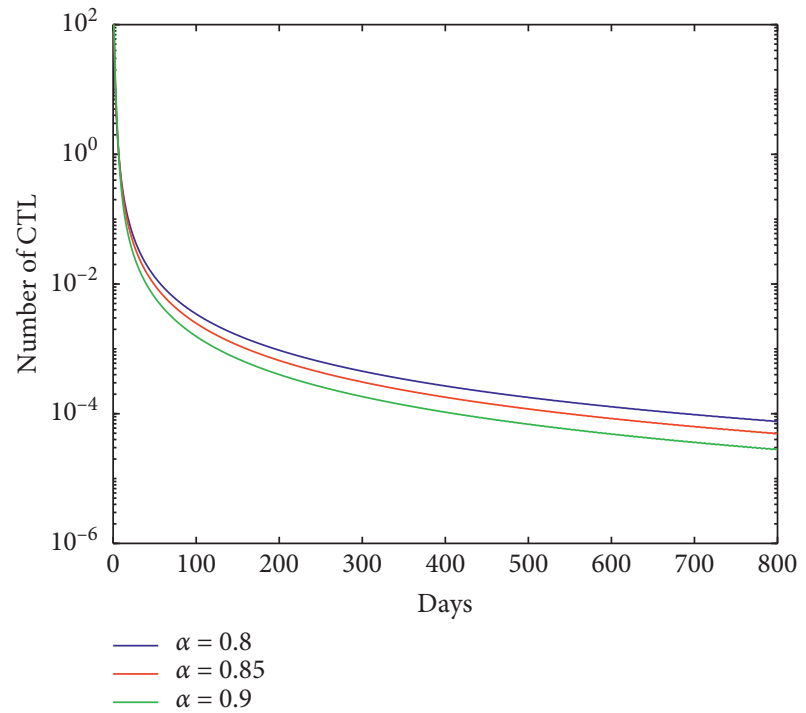

(b)

Figure 3: Continued. 


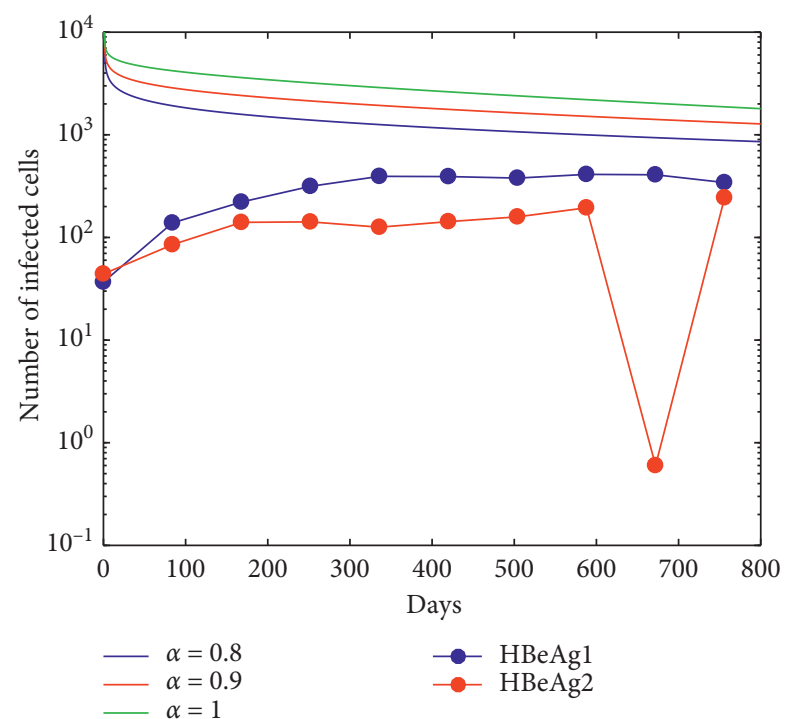

(c)

FIGURE 3: Simulation results of successful-treatment group.

different change rate with different $\alpha$, and the positive correlation between log-infected cells and log-HBeAgis reflected roughly. The positive correlation is not so good for patient 2's HBeAg data, but the trend is consistent between the infected cells and HBeAg. We can see there also exists some difference with different $\alpha$ which may reflect the individual difference.

\section{Conclusions}

In this paper, based on the fact that immune response has memory, we discussed a fractional-order HBV model with standard mass action incidences, and we obtained the basic reproductive numbers $R_{0}$ and the cytotoxic $\mathrm{T}$ lymphocytes' immune-response reproductive number $R_{1}$. When $R_{0}<1$, we have proved that $E_{0}$ is globally asymptotically stable which meant the infected person can eventually recover automatically even when infected with a large number of HBV. When $R_{0}>1$ and $R_{1}<1, E_{1}$ was locally asymptotically stable which meant the one infected by HBV with no immune response would be infected persistently. When $R_{1}>1$, we also gave the local stable condition of $E_{2}$, which meant the infected person has immune response to $\mathrm{HBV}$, but the persistent infection still existed. Furthermore, we gave some simulations with different $\alpha$ to test our theoretical results and some clinical phenomena. The simulation showed that our model can simulate the positive correlation between loginfected cells and log-HBeAg to some extent. On the other hand, even with the same initial conditions and the same parameters, we also found that there existed some difference in the dynamic routes with different order $\alpha$, which may reflect the individual difference. From the analysis and simulation, we can see the fractional-order model maybe more reasonable to describe HBV immune course.

\section{Discussion}

In our paper, we found that the fractional-order model can reflect the characteristics of immune memory and clinical individual differences, but it should be pointed out that there are only three variables in our model; the model only described HBV infection roughly. Furthermore, we could not obtain the number of uninfected cells, infected cells, and CTL; when we performed the simulation, we only chose the parameters from biological meaning, not from real clinical data, so there are still many works to be done in the future.

\section{Data Availability}

The HBeAg data used to support the findings of this study are included within the article.

\section{Conflicts of Interest}

The authors declare that there are no conflicts of interest regarding the publication of this paper.

\section{Acknowledgments}

This study was jointly supported by 2015 National Traditional Medicine Clinical Research Base Business Construction Special Topics (JDZX2015299) and Fundamental Research Funds for the Central Universities (FRF-BR-16019A).

\section{References}

[1] Y. Xiao, X. Xu, and S. Tang, "Sliding mode control of outbreaks of emerging infectious diseases," Bulletin of Mathematical Biology, vol. 74, no. 10, pp. 2403-2422, 2012.

[2] Y. Zheng, L. Q. Min, Y. Ji, Y. Su, and Y. Kuang, "Global stability of endemic equilibrium point of basic virus infection 
model with application to HBV infection," Journal of Systems Science and Complexity, vol. 23, no. 6, pp. 1221-1230, 2009.

[3] G. Huang, W. Ma, and Y. Takeuchi, "Global properties for virus dynamics model with Beddington-DeAngelis functional response," Applied Mathematics Letters, vol. 22, no. 11, pp. 1690-1693, 2009.

[4] G. Huang, H. Yokoi, Y. Takeuchi, T. Kajiwara, and T. Sasaki, "Impact of intracellular delay, immune activation delay and nonlinear incidence on viral dynamics," Japan Journal of Industrial and Applied Mathematics, vol. 28, no. 3, pp. 383411, 2011.

[5] T. Q. Zhang, J. L. Wang, Y. Li, Z. Jiang, and X. F. Han, "Dynamics analysis of a delayed virus model with two different transmission methods and treatments," Advances in Difference Equations, vol. 2020, no. 1, pp. 1-17, 2020.

[6] W. Wang, W. B. Ma, and Z. S. Feng, "Dynamics of reaction-diffusion equations for modelling $\mathrm{CD}^{+} \mathrm{T}$ cells decline with general infection mechanism and distinct dispersal rate," Nonlinear Analysis: Real World Applications, vol. 51, no. 2, Article ID 102976, 2020.

[7] C. Yan and W. Wang, "Modeling HIV dynamics under combination therapy with inducers and antibodies," Bulletin of Mathematical Biology, vol. 81, no. 7, pp. 2625-2648, 2019.

[8] R. Q. Shi, T. Lu, and C. H. Wang, "Dynamic analysis of a fractional order delayed model for hepatitis B virus with CTL immune response," Virus Research, vol. 277, no. 2, Article ID 197841, 2020.

[9] S. He, S. Y. Tang, S. Tang, and L. Rong, "A discrete stochastic model of the COVID-19 outbreak: forecast and control," Mathematical Biosciences and Engineering, vol. 17, no. 4, pp. 2792-2804, 2020.

[10] A. S. Perelson, "Modelling viral and immune system dynamics," Nature Reviews Immunology, vol. 2, no. 1, pp. 28-36, 2002.

[11] M. A. Nowak, S. Bonhoeffer, A. M. Hill, R. Boehme, H. C. Thomas, and H. McDade, "Viral dynamics in hepatitis B virus infection," Proceedings of the National Academy of Sciences, vol. 93, no. 9, pp. 4398-4402, 1996.

[12] G. K. Lau, M. Tsiang, J. Hou et al., "Combination therapy with lamivudine and famciclovir for chronic hepatitis B-infected Chinese patients: a viral dynamics study," Hepatology, vol. 32, no. 2, pp. 394-399, 2000.

[13] L. Zhang, G. Huang, A. Liu, and R. Fan, "Stability analysis for a fractional HIV infection model with nonlinear incidence," Discrete Dynamics in Nature and Society, vol. 2015, Article ID 563127, 11 pages, 2015.

[14] A. A. M. Arafa, S. Z. Rida, and M. Khalil, "A fractional-order model of HIV infection with drug therapy effect," Journal of the Egyptian Mathematical Society, vol. 22, no. 3, pp. 538-543, 2014.

[15] Y. Wang, L. Liu, X. Zhang, and Y. Wu, "Positive solutions of an abstract fractional semipositone differential system model for bioprocesses of HIV infection," Applied Mathematics and Computation, vol. 258, pp. 312-324, 2015.

[16] Y. Yan and C. Kou, "Stability analysis for a fractional differential model of HIV infection of $\mathrm{CD}^{+}{ }^{+} \mathrm{T}$-cells with time delay," Mathematics and Computers in Simulation, vol. 82, no. 9, pp. 1572-1585, 2012.

[17] S. M. Salman and A. M. Yousef, "On a fractional-order model for HBV infection with cure of infected cells," Journal of the Egyptian Mathematical Society, vol. 25, no. 4, pp. 445-451, 2017.

[18] L. C. Cardoso, F. L. P. Dos Santos, and R. F. Camargo, "Analysis of fractional-order models for hepatitis B,"
Computational and Applied Mathematics, vol. 37, no. 4, pp. 4570-4586, 2018.

[19] Y. Su, L. Zhao, and L. Min, "Analysis and simulation of an adefovir anti-hepatitis B virus infection therapy immune model with alanine aminotransferase," IET Systems Biology, vol. 7, no. 5, pp. 205-213, 2013.

[20] K. Wang, W. Wang, H. Pang, and X. Liu, "Complex dynamic behavior in a viral model with delayed immune response," Physica D: Nonlinear Phenomena, vol. 226, no. 2, pp. 197-208, 2007.

[21] B. Z. Guo and L. M. Cai, "A note for the global stability of a delay differential equation of hepatitis B virus infection," Mathematical Biosciences \& Engineering, vol. 8, no. 3, pp. 689-694, 2012.

[22] D. Tavares, R. Almeida, and D. F. M. Torres, "Caputo derivatives of fractional variable order: numerical approximations," Communications in Nonlinear Science and Numerical Simulation, vol. 35, pp. 69-87, 2016.

[23] Y. Emvudu, D. Bongor, and R. Koïna, "Mathematical analysis of HIV/AIDS stochastic dynamic models," Applied Mathematical Modelling, vol. 40, no. 21-22, pp. 9131-9151, 2016.

[24] E. Ahmed, A. M. A. El-Sayed, and H. A. A. El-Saka, "On some Routh-Hurwitz conditions for fractional order differential equations and their applications in Lorenz, Rössler, Chua and Chen systems," Physics Letters A, vol. 358, no. 1, pp. 1-4, 2006.

[25] J. Huo, H. Zhao, and L. Zhu, "The effect of vaccines on backward bifurcation in a fractional order HIV model," Nonlinear Analysis: Real World Applications, vol. 26, pp. 289-305, 2015.

[26] M. Çiçek, C. Yakar, and B. Oğur, "Stability, boundedness, and Lagrange stability of fractional differential equations with initial time difference," The Scientific World Journal, vol. 2014, Article ID 939027, 2014.

[27] W. J. Yin, F. Wang, L. P. Shen et al., “The positive correlation of log hepatitis B virus (HBV) deoxyribonucleic acid load and $\log \mathrm{HBeAg}$ and $\log$ antibody to HBeAg," Chinese Journal of Vaccines and Immunization, vol. 18, no. 4, pp. 316-319, 2012.

[28] L. Q. Min, Y. M. Su, and Y. Kuang, "Mathematical analysis of a basic virus infection model with application to HBV infection," Rocky Mountain Journal of Mathematics, vol. 38, no. 5, pp. 1573-1585, 2008.

[29] M. Hellerstein, M. B. Hanley, D. Cesar et al., "Directly measured kinetics of circulating T lymphocytes in normal and HIV-1-infected humans," Nature Medicine, vol. 5, no. 1, pp. 83-89, 1999. 